\title{
Burundi Population Awareness about Gout and the Pivotal Role Played by Physical Exercise in Prevent and Managing Gout Relating Problems
}

\section{NGAYIMBESHA Adrien", BIZIMANA Jean Berchmans and GAKIMA Marie Stella}

Institute of Physical Education and Sports, University of Burundi, Burundi

*Corresponding author: NGAYIMBESHA Adrien, Institute of Physical Education and Sports, University of Burundi, Burundi

\begin{abstract}
Introduction: Prevalence and incidences of gout disease had shown an increasing trend all over the world including in developing countries. In underdeveloped countries like Burundi, there is no information about this disease situation, and the population may lack sufficient knowledge to prevent this metabolic pathology.
\end{abstract}

Study objective: The present study was aimed to assess Burundi population level of knowledge on gout.

Method: A self-report questionnaire method has been used in data collection. Participants have been recruited in a sport club.

Results: According to the study result's, on all selected items, Burundi population presents inadequate knowledge on gout disease.

\section{Keywords}

Gout disease, Burundi population awareness, Physical exercise

\section{Introduction}

Gout is a type of inflammatory arthritis that may sometimes be called gouty arthritis. Gout develops in some people who have high levels of uric acid in their body and bloodstream, a condition called hyperuricemia. When uric acid builds up in the joints, it can form needle-like crystals. This can cause inflammation and sudden and severe pain, as well as stiffness, tenderness, redness, warmth and swelling. The pain may last hours or weeks and make it difficult to perform daily activities. The build-up of uric acid can look like lumps under the skin, called tophi. It can also collect in the kidneys and cause kidney stones (small, hard deposits).

This metabolic problem is the most prevalent type of chronic inflammatory arthritis in adults [1]. Symptoms produced by elevated uric acid levels are pain and swelling of the Big toe, painful joint especially the knee joint. Many patients even remain symptom free despite of high uric acid levels patients with gout alone or combined with renal stones should be distinguished prior to treatment. Abnormalities in serum uric acid metabolism may cause hyperuricemia and gout. Hyperuricemia is the result of interactions among multiple factors, including sex, age, genetics, lifestyle, and environment [2].

Several studies have suggested that hyperuricemia is associated with many diseases, including diabetes mellitus [3], hypertension [4,5], stroke [6,7], dyslipidemia [8], chronic kidney disease [9], cardiovascular events, and heart failure [10].

Gout is also a serious health issue and is an independent risk factor for heart failure and metabolic syndrome [11]. Consider its negative impact on quality of life, gout constitute a serious public health problem that need to be solved. In many developed countries, studies have shown this disease prevalence and incidences $[12,13]$. In my country (Burundi), there is no published study on gout evolution situation or on the population awareness and knowledge on this threatening public health problem. However, even though we lack information on this disease in Burundi, we can speculate that in urban and sub-urban area, gout dis- 
Table 1: Participant's characteristics.

\begin{tabular}{|l|l|l|}
\hline Characteristics & Mean & SD \\
\hline Age & 42 & \pm 11 \\
\hline Weight $(\mathrm{kg})$ & 68 & \pm 13 \\
\hline
\end{tabular}

ease does exist in urban population. Therefore, study on this disease tendency and population knowledge on it is very important. Hence, in the current study, we intend to know Burundi population knowledge on gout etiology, and how they can themselves prevent this.

\section{Method}

\section{$1^{0}$ Study participants}

Participant to the study was recruited among ISHAKA Jogging club, doing physical activity once a week (Saturday). Prior to questionnaire distribution, we have taken moment to explain to our study participants the aim of the study and anonymous of their responses. A total of 25 persons accepted to respond to prepared questionnaire. Below Table 1 show characteristics of our study subject.

\section{$2^{0}$ Study design}

The current study was a descriptive study to assess the knowledge and awareness of Burundi population on gout problem: General knowledge, risk factors of gout and related food to avoid or to limit in case of gout to prevent this.

Once the study approved by the Ethics Committee of the Faculty of medicine of Burundi national university, strategy to conduct the study has been developed.

The group of participants has been approached and participation acceptation has been obtained. The self-report questionnaire for each participant to the study was distributed during the day of this club sport (Saturday).

After collecting back completed questionnaire the researcher grouped the data into different subdivision of questionnaire after which the data were analyzed.

\section{$3^{0}$ Data collection}

Information about population knowledge on gout disease has been obtained using self-report questionnaire method. The used questionnaire was developed by us. A pilot study was organized in way to explore any possible confusion or ambiguity during completion with the ultimately goal of determination of questionnaire validity. Questionnaire amendments were then made according to pilot study comments. Used questionnaire was divided into:

$\checkmark$ General knowledge on gout symptoms (5 questions)

$\checkmark$ Risk factors for gout (8 questions)

$\checkmark$ Food to avoid or limit in case of gout (7 questions) $\checkmark$ Way physical activity improve gout symptoms (10 questions)

\section{$4^{0}$ Scoring}

For the theoretical knowledge, one point was given for correct answer and zero for incorrect answer. Thus, a Total of 30 points were allotted under knowledge. To interpret the level of knowledge, the score was distributed as: $\leq 50 \%$ inadequate knowledge; 51-69 moderately adequate knowledge and $\geq 70$ adequate knowledge.

\section{$5^{0}$ Statistical analysis}

Due to the nature of the study (descriptive study), descriptive statistics was mainly used in data analysis and discussion. Frequency and percentage distribution were used to analyze data and level of knowledge of Burundi population regarding gout disease.

\section{$5^{0}$ Ethical consideration}

The ethic committee of the faculty of medicine within the Burundi University approved the study method and protocol.

\section{Results}

Obtained results of the present study have been presented in the following headings:

-Scoring results on population Theoretical knowledge on gout disease;

-Frequency and percentage distribution of knowledge among our study participants.

Results contained in above Table 2 show that our study participants have a poor knowledge in all items selected to evaluate their knowledge regarding gout disease.

Statistical results show that our questioned participants have obtained a mean score of 1.7 in general knowledge on gout symptoms, with a standard deviation of 0.232 . Regarding to risk factor for gout, our participants have scored 2.3, with a standard deviation of 1.121. Testing their knowledge on food to avoid or to limit in case of gout, participant to the study obtained a mean score of 2.7 with a standard deviation of 0.923 . Physical exercise constitutes one way among others used to prevent or manage gout problem. The present study results show that our participants are unknowledgeable on this. The mean score obtained by this study is 3.8 with a standard deviation of 1.420 . For overall items, participants have scores 10.5 with a standard deviation of 0.523 .

To qualify our participant's level of knowledge regarding gout disease, obtained scores were distributed as: $\leq 50 \%$ inadequate knowledge; 51-69 moderately adequate knowledge and $\geq 70$ adequate knowledge.

Statistical results contained in Table 3 demonstrat- 
Table 2: Scoring results on gout theoretical knowledge.

\begin{tabular}{|l|l|l|l|l|}
\hline Knowledge variables & Maximum & Mean & Mean percentage & Standard deviation \\
\hline General knowledge on gout symptoms & 5 & 1.7 & 34 & \pm 0.232 \\
\hline Risk factors for gout & 8 & 2.3 & 28.75 & \pm 1.121 \\
\hline Food to avoid or limit in case of gout & 7 & 2.7 & 38.57 & \pm 0.923 \\
\hline Way physical activity improve gout symptoms & 10 & 3.8 & 38 & \pm 1.420 \\
\hline Overall Knowledge score max & $\mathbf{3 0}$ & $\mathbf{1 0 . 5}$ & $\mathbf{3 5}$ & $\mathbf{0 . 5 2 3}$ \\
\hline
\end{tabular}

Table 3: Frequency and percentage distribution of knowledge among our participants.

\begin{tabular}{|c|c|c|c|c|c|c|}
\hline \multirow[b]{2}{*}{ Knowledge variables } & \multicolumn{2}{|c|}{ Inadequate $\leq \mathbf{5 0} \%$} & \multicolumn{2}{|c|}{ Moderately adequate $51-69 \%$} & \multicolumn{2}{|c|}{ Adequate $\geq 70 \%$} \\
\hline & $\mathbf{N}$ & $\%$ & $\mathbf{N}$ & $\%$ & $\mathbf{N}$ & $\%$ \\
\hline General knowledge on gout symptoms & 19 & 76 & 6 & 24 & - & - \\
\hline Risk factors for gout & 18 & 72 & 6 & 24 & 1 & 4 \\
\hline Food to avoid or limit in case of gout & 21 & 84 & 4 & 16 & - & - \\
\hline Way physical activity improve gout symptoms & 16 & 64 & 7 & 28 & 2 & 8 \\
\hline
\end{tabular}

ed that big majority of the present study participant has inadequate knowledge on gout disease. In fact, $76 \%$ participants presented inadequate knowledge on gout symptoms against $24 \%$ with moderately adequate knowledge. No one among participant to the study presents adequate knowledge on gout symptoms. Similar results have been obtained when asking our participants risk factors for gout. On this item of questions, we observed that $72 \%$ of study participant have inadequate knowledge on gout risk factors, while $24 \%$ among them possess a moderately adequate knowledge on this. An insignificant number of participant $(\mathrm{N}=4)$ are adequately knowledgeable on risk factors of gout disease.

Assessing our participant level of knowledge on food to avoid or to limit in case of gout or in prevent of this disease, obtained statistical results show that a highly significant number of participant to the present study present poor knowledge (in adequate knowledge) on adequate diet to take in prevention or in managing gout disease. This has been obtained at a level of $84 \%$ among participant. No one among our subjects is adequately knowledgeable on food to avoid or to limit in case of gout problem. A very few participants (8\%) to the present study now how exercise and physical activity aid in gout management. This show that majority of our subject are not adequately knowledgeable on the role of exercise and physical activity in the prevention and management of gout problem.

\section{Discussion}

The aim of the present study was to evaluate Burundi population level of knowledge and awareness on gout disease. Results provides by the current study show that participant to the present study possess a poor theoretical knowledge on gout disease. In fact, according to statistical results, $76 \%$ of our study subject has been classified in inadequate knowledge category when consider their knowledge in term of gout symptoms. To this selected knowledge, only $23 \%$ of partic- ipants agree that the signs and symptoms of gout almost always suddenly and acutely, $41 \%$ agree that gout cause acute pain in joints, $35 \%$ agree that gout cause redness and swelling of the joint, and $9 \%$ of participants agree that a gout attack can last for 5 to 10 days. A low percentage of participants (13\%) know that can attack toes extremity.

Participant to the present study have been questioned on etiology and predisposition factors to assess their level of knowledge on gout disease.

Similarly, to their knowledge results on gout symptoms, participant showed a very weaken knowledge on etiology and predisposition factors of gout. On all question's items used to assess their etiology and predisposition knowledge on gout only $21 \%$ were well knowledgeable to this. Take example on some items among others used to assess participant knowledge on gout symptoms and etiology, about $87 \%$ of subject doesn't know that hypertension increase the risk of gout disease; $91 \%$ of participant to this study doesn't know that hyperinsulinemia increase also the risk of gout. Also $88 \%$ of participant to the present study failed to correctly respond that diabetes mellitus constitutes another risk factor of gout disease. Obtained results to the assed knowledge (gout symptoms and etiology) are quite similar to [14] results. In their study carried out in Qatar on Qatari population knowledge on gout disease, they found that only 31\% of Qatari population was well knowledgeable on symptoms and etiology of gout disease. Other studies findings have indicated that diabetes, hypertension, hyper insulenemia, obesity, elevated triglyceride and cholesterol levels were all associated with the risk of gout [15]. However, existing literature has not totally elucidated the mechanism by which excess body fat leads to increased serum uric acid. The probable mechanism by which fat influence gout is that accumulation visceral fat leads to an increase in free fatty acids and tumor 
necrosis factor alpha (TNF- $\alpha$ ), together with a decrease in adiponectin concentration, which leads in turn to reduced renal excretion of uric acid [16].

In this study, a lack of awareness regarding association between dietary and gout disease is confirmed by the present study results. A big majority (97\%) of the present study participant responded that see food consumption doesn't increase the risk of gout attack, more than half of the present study participant (61\%) know that beef, pork meat and others consumption increase the risk of gout disease. We also observed through the present study results that non-negligible participant (53\%) believe that intake of vegetables was associated with a risk of gout disease. However, in the literature, it is well confirmed that there is an increased risk of gout with higher levels of beef, pork and seafood consumption [17]. It is also proved that moderate consumption of purine-rich vegetables has not been associated with an increased risk of gout [18].

Change way of life such as avoiding sedentarily constitutes one way of preventing or managing gout disease. Unexpectedly, over than $60 \%$ of our study subjects ignore this. Only $8 \%$ of our participant to the study adequately knows that physical activity can help in preventing and managing gout problem. Globally, obtained results show that participant to the study lack of knowledge on gout disease. This means that Burundi population is not educated to gout disease. As gout is common disease with significant impact on quality of life, and has an increased risk of mortality, population education on this is for great importance. Patient education must constitute an integral part in prevention, managing, treating early arthritis and ankylosing spondylitis.

During the last decades there has been an ongoing development within healthcare, moving away from the view of health professionals as the only experts and providers of knowledge and patients as passive recipients towards a more collaborative approach. Patients have been recognized as active agents in managing their illness and own health care [19]. Population or patient education comprises all educational activities provided for patients, including aspects of therapeutic education, health education and health promotion [20].

The principle of "shared decision making" allows patients and their providers to make healthcare decisions together, based on the best scientific evidence available, as well as the patient's values and preferences, is increasingly accepted [21].

The main goal of population and/or patient education is not only the knowledge transfer and disease control, but also to enable patients to manage their illness, adjust to their condition and maintain quality of life [22].

\section{Conclusion}

The study found that Burundi population knowledge and awareness on gout disease is inadequate. Population lack sufficient knowledge on risk factors, sign and symptoms, and food to avoid when already developed this disease.

Burundi population education on gout disease through campaign, media and social media, seminars is needed to enhance and promote public awareness on this disease. This education may focus on gout risk factors, sign and symptoms and predisposition factors.

\section{References}

1. Zhu Y, Pandya BJ, Choi HK (2011) Prevalence of gout and hyperuricemia in the US general population: the National Health and Nutrition Examination Survey 2007-2008. Arthritis Rheum 63: 3136-3141.

2. B L, T W, Hn Z, Ww Y, Hp Y, et al. (2011) The prevalence of hyperuricemia in China: a meta-analysis. BMC Public Health 11: 832.

3. Nakanishi N, Okamoto M, Yoshida H, Matsuo Y, Suzuki K, et al. (2003) Serum uric acid and risk for development of hypertension and impaired fasting glucose or Type II diabetes in Japanese male office workers. Eur J Epidemiol 18: $523-530$.

4. Johnson RJ, Kang DH, Feig D, Kivlighn S, Kanellis J, et al. (2003) Is there a pathogenetic role for uric acid in hypertension and cardiovascular and renal disease? Hypertension 41: 1183-1190.

5. Wang J, Qin T, Chen J, Li Y, Wang L, et al. (2014) Hyperuricemia and risk of incident hypertension: a systematic review and meta-analysis of observational studies. PLoS One 9: e114259.

6. Li M, Hou W, Zhang X, Hu L, Tang Z (2014) Hyperuricemia and risk of stroke: a systematic review and meta-analysis of prospective studies. Atherosclerosis 232: 265-270.

7. Bos MJ, Koudstaal PJ, Hofman A, Witteman JC, Breteler MM (2006) Uric acid is a risk factor for myocardial infarction and stroke: the Rotterdam study. Stroke 37: 1503-1507.

8. Peng TC, Wang CC, Kao TW, Chan JYH, Yang YH, et al. (2015) Relationship between hyperuricemia and lipid profiles in US adults. BioMed Research International 2015: 7.

9. Chang HY, Tung CW, Lee PH, Lei CC, Hsu YC, et al. (2010) Hyperuricemia as an independent risk factor of chronic kidney disease in middleaged and elderly population. Am J Med Sci 339: 509-515.

10. Huang H, Huang B, Li Y, Huang Y, Li J, et al. (2014) Uric acid and risk of heart failure: a systematic review and metaanalysis. Eur J Heart Fail 16: 15-24.

11. Annemans L, Spaepen E, Gaskin M, Bonnemaire M, Malier V, et al. (2008) Gout in the UK and Germany: prevalence, comorbidities and management in general practice 20002005. Ann Rheum Dis 67: 960-966.

12. Doherty M (2009) New insights into the epidemiology of gout. Rheumatology 48: 2-8.

13. Liu R, Han C, Wu D, Xia X, Gu J, et al. (2015) Prevalence of Hyperuricemia and Gout in Mainland China from 2000 to 2014: A Systematic Review and Meta-Analysis. Biomed Res Int 2015: 762820.

14. Mohammed I Alshammari M, Ali Mujtaba Md (2017) Public Knowledge and Awareness about Gout: A Cross-sectional Study in Qatar. Journal of Pharmaceutical Research International 17: 1-11. 
15. Choi HK, Atkinson K, Karlson EW, Curhan G (2005) Obesity, weight change, hypertension, diuretic use, and risk of gout in men: The health professional's follow-up study. Arch Intern Med 165: 742-748.

16. Choi HK, Mount DB, Reginato AM; American College of Physicians; American Physiological Society (2005) Pathogenesis of gout. Ann Intern Med 143: 499-516.

17. Zhang $\mathrm{Y}$, Chen $\mathrm{C}$, Choi $\mathrm{H}$, Chaisson $\mathrm{C}$, Hunter D, et al. (2012) Purine-rich foods intake and recurrent gout attacks. Ann Rheum Dis 71: 1448-1453.

18. Harrold LR, Kathleen MM, Daniel P, Nausheen N, Cassandra F, et al. (2012) Patients' knowledge and beliefs concerning gout and its treatment: a population-based study. BMC Musculoskelet Disord13: 180.
19. Hoving C, Visser A, Mullen PD, van den Borne B (2010) A history of patient education by health professionals in Europe and North America: from authority to shared decision making education. Patient Educ Couns 78: 275281.

20. Albano MG, Giraudet-Le Quintrec JS, Crozet C, dlvernois JF (2010) Characteristics and development of therapeutic patient education in rheumatoid arthritis: analysis of the 2003-2008 literature. Joint Bone Spine 77: 405-410.

21. Chewning B, Bylund CL, Shah B, Arora NK, Gueguen JA, et al. (2012) Patient preferences for shared decisions: a systematic review. Patient Educ Couns 86: 9-18.

22. de Ridder D, Geenen R, Kuijer R, van Middendorp H (2008) Psychological adjustment to chronic disease. Lancet 372 : 246-255. 